\title{
HOW TO EVALUATE AND ASSESS QUALITY OF LIFE ISSUES IN HEAD AND NECK CANCER PATIENTS
}

Jose Guilherme Vartanian, MD, PhD'; Simon N. Rogers, MD²; Luiz Paulo Kowalski, $\mathrm{MD}, \mathrm{PhD}^{1}$

${ }^{1}$ Head and Neck Surgery and Otorhinolaryngology Department, A. C. Camargo Cancer Center, Sao Paulo, Brazil

${ }^{2}$ Evidence-Based Practice Research Centre (EPRC), Faculty of Health and Social Care, Edge Hill University, Ormskirk, UK; Consultant Regional Maxillofacial Unit, University Hospital Aintree, Liverpool, UK

Author of correspondence:

Jose G. Vartanian, MD, PhD

Rua Professor Antonio Prudente, 211, Liberdade, Sao Paulo, Brazil

Phones: +55 11 2189-5172 / +55 11 98381-3032

Email: jgvartanian@uol.com.br 


\begin{abstract}
Purpose of review: Review quality of life (QOL) concepts and most common instruments to be used in patients with head and neck cancer, as well as the potential benefits and limitations of information derived from QOL studies.
\end{abstract}

Recent findings: Information from QOL studies can be clinical predictors of prognosis, serve as potential screening and planning tools for clinical care and rehabilitation efforts. Enhancements in computer technology and the advent of tools like HNC specific item prompt list will allow for QOL data to be used more easily.

Summary: Patients with malignant head and neck neoplasms can present changes in important vital functions related to the disease and its treatment, usually resulting in a negative impact on their QOL. The application of specific questionnaires can be used to measure such impact and the information derived from QOL studies has the potential to be incorporated in the clinical practice to improve the quality of care.

Keywords: quality of life; head neck neoplasm; questionnaires 


\section{Introduction}

Since the middle of last century, when the World Health Organization (WHO) extended the definition of health beyond "absence of disease or infirmity" to include "a state of physical, mental and social well-being", there has been an increasing interest to study topics related to the functional, psychological and social aspects of individuals, and many of these concepts are related to what we now call quality of life (QOL) [1,2]. Currently QOL evaluation has been recognized as an important outcome measure in medicine, mainly in chronic diseases scenarios.

The evaluation of the QOL in patients with cancer is essential, considering not only the chronic nature of the disease but also the psychological impact of the disease and the treatment on the individual since its diagnosis, which also ends up being reflected in the familial, social and professional life.

Head and neck cancer is a worldwide public health problem, mainly in developing countries where most cases are diagnosed at advanced stages. In such patients, despite the use of more aggressive and multimodal treatment approaches and better reconstruction and multidisciplinary reahabilitation care, the treatment can result in significant aesthetic and functional sequelae as well as in a poor survival rate [3].

Patients with malignant head and neck neoplasms, due to the location, stage and treatment of these lesions, can present changes in some vital functions as chewing, swallowing, speech, appearance, as well as loss of taste, chronic pain in the shoulder, among others. As a consequence, these sequelae can alter important functions related to the feeding, communication and social interaction of individuals, which may affect their self-esteem, limit their activities and their capacity for work, and restrict the relations of individuals with their relatives and friends, usually resulting in a negative impact on the QOL of these patients $[3,4]$.

In addition to these factors, with recent advances in the diagnostic procedures, advances in surgery (mainly related to a less invasive techniques such as robotic and endoscopic approaches and improvements in microvascular reconstruction), advances in radiation and chemotherapy options, we currently have different therapeutic modalities for the treatment of some tumor subsets, 
with expectation of similar survival rates but with different functional and aesthetic results. This makes QOL assessment in such individuals a useful tool to help in the planning of treatment strategy. A practical example would be the treatment of patients with initial glottic tumors, where exclusive radiotherapy, open partial laryngectomies or the endoscopic surgical approaches with laser are all valid and feasible options with similar curative potential but can generate different functional as well as different aesthetic results.

In this context, evaluation of QOL is an important tool for understanding the impact of the head and neck cancer and its treatment on the patient's life, as well as it could be a valuable tool to better plan the treatment and rehabilitation strategies $\left[3,4,5^{* *}, 6\right]$.

\section{QOL definition and role}

There are various difficulties involved in QOL studies. First of all, despite numerous studies, there is still no universally accepted definition of quality of life. According to the World Health Organization (WHO), quality of life is defined as "the individual's perception of their position in life in the context of the culture and value systems in which they live and in relation to their goals" [7]. This concept was well defined by Morton and Izzard defining QOL as the "perceived discrepancy between the reality of what a person has and the concept of what the person wants, needs, or expects" [8]. However, as several factors associated to QOL are not directly related to health and medical conditions, QOL studies in medicine usually are focused on aspects associated to the effect of a disease and its treatment on patient's live, known as health-related QOL (HRQOL) [9**].

The role of QOL assessment in patients with HNC are linked to several important aspects related to treatment decision, physical and functional rehabilitation directioning, social and emotional support and in the evaluation of treatment outcomes. Such evaluation also can play a role as primary or secondary outcome measure of clinical trials [3-5**].

In many clinical situations, when the there is a clear tumor control and survival difference in favor of one treatment, it must be the treatment of choice, in spite of the risk of functional and aesthetical sequelae. Several approaches using 
reconstructive methods and multidisciplinary care must be used aiming to reduce the undesirable treatment consequences. So, in this situation, quality of life do not influence treatment decision, but it stimulates the creativity for new rehabilitation methods. On the other hand, results of QOL evaluation in HNC patients in the treatment decision-making process can be considered as a major role when two different treatments have similar chance of cure but have different QOL outcomes and also in non-curative (palliation) treatment consideration [10]. We can also list some areas where information derived from QOL studies could change treatment planning: use of IMRT, organ-preservation scenarios, HPV+ related oropharyngeal cancer, neck dissection, minimally invasive approaches, reconstruction after major surgeries, and directing supportive management after treatment $\left[4,5^{* *}, 10\right]$.

In summary, information achieved by QOL studies can help identify and better describes the effects of the disease and its treatment on patients' lives. It can also result in changes in therapeutic, rehabilitation and social support procedures, as well as assisting the physician and patient in the therapeutic decision. An important issue is that QOL assessment is based on what is important and matters to patients, and provides evidence-based information that can be used to improve the quality of health care practices [11].

\section{How to evaluate QOL - General considerations}

There are several methods to evaluate QOL in HNC patients. Qualitative approaches such as semi-structured interviews provide the opportunity for a better understanding of patient experience, but usually are more demanding and time consuming, and the results tend to be descriptive. The majority of QOL studies are performed through the application of specific questionnaires, developed for this purpose. In most cases, the development of these questionnaires usually includes a commission of specialists in the area, some of them include patient participation, following internationally established methodology to guarantee their psychometric properties $[9 * *, 12]$.

Generally, QOL assessment questionnaires are composed of several questions with response options that may be "YES-NO", response categories on scales (ranging from 3 to 7 options or points - Likert scales), or visual analogue 
scales (VAS). These issues are aggregated into "domains" or "dimensions", together with a global QOL score. It is important to attempt to identify scores that reflect a clinically meaningful difference in QOL outcome. This might be different to scores that reach statistical significance. For example a 10-point difference in the subscales of the UW-QOL is deemed to imply clinical importance [9**]. This is essential when considering how to best interpret and implement the results of such studies into clinical daily practice [13**]. The results of the obtained scores should preferably be reported in a format that may be clinically relevant and easy to be understood. In general, one can interpret the results by comparing them with values called "anchors", that is, by comparing the scores of patients with different objective functional states ("anchors") (for example, patients who have a full oral diet usually have a "x" score, and those who eat exclusively with liquids have a "y" score; tracheostomized patients usually have " $z$ " scores; etc), or interpret the results by statistical calculations based on the distribution of values, also called minimal clinically important difference [14].

In general, QOL questionnaires can be divided into three groups; generic questionnaires, disease-specific questionnaires, and symptom or treatment specific questionnaires. Generic questionnaires aim to assess the QOL of individuals independent of the disease in question, providing an overall assessment of the patient's functional status and well-being. Disease-specific questionnaires are designed to evaluate the quality of life related to the main aspects that may be being altered specifically by a particular disease and its treatment. Symptom or treatment specific questionnaires, designed to assess the QOL related to a specific symptom or specific treatment modality. Disease-specific questionnaires are considered to be more precision, less influenced by comorbidities, and more sensitive to clinical changes over time $\left[9^{* *}, 13^{* *}\right]$. A combination of global evaluation and a head and neck specific or functional questionnaire gives a broader reflection of patient reported outcome. However, such evaluations would be considered long, costly and could overburden the individuals making it difficult to be accepted and difficult to be used in daily practice.

Thus, the evaluation of QOL in patients with head and neck cancer is usually performed through the application of specific questionnaires, being considered an 
effective method to evaluate the therapeutic results regarding the physical, functional and psychosocial aspects. The choice of such instrument should be hypothesis-driven, fitting the study's purpose.

\section{Instruments for QOL evaluation in HNC patients}

Several questionnaires have already been proposed to evaluate the QOL in the population of patients with head and neck cancer. Although there is no goldstandard instrument, several authors recommend that the ideal instrument should be short, concise, easy to understand, self-applied by patients to reduce health professional interference, have low cost, minimum expenditure of time for completion and that has its psychometric validation criteria well established. The questionnaires most commonly used to assess quality of life in patients with head and neck cancer were the EORTC QLQ-H \& N35 questionnaires, followed by the University of Washington (UW-QOL) and FACT-H\&N $\left[9^{* *}, 13^{* *}\right]$.

Handle on QOL is a unique online searchable database of all papers published from 1982 to present on quality of life in head and neck cancer that have used questionnaires [15**]. The site has over 1325 papers and reveals that over 250 different questionnaires have been used in studies reported QOL in head and neck cancer. This emphasizes the complexity of the potential multiple influences in QOL following HNC, the challenges of such assessment, and the scope for further research. In a systematic review publication about QOL instruments used in HNC patients, the authors identified that in 710 articles selected for the review, there were a total of 57 different QOL instruments used [13**]. Such article highlighted the questionnaires available to be used in HNC patients, and subdivided the instruments in three groups to facilitate the selection for future studies: site (head and neck)-specific, treatment-specific and symptom-specific survey instruments. Examples of treatment-specific measures include: QOL radiation therapy index (QOL-RTI), the Neck Dissection Impairment Index (NDI), and the Shoulder Pain and Disability Index. Examples of symptom-specific measures include: MD Anderson Dysphagia Inventory (MDADI), the Xerostomia Questionnaire, Voice Handicap Index (VHI), and the Derriford Appearance Scale (DAS24). 
Given the complexity of QOL evaluation and the many physical, functional and emotional elements that can be affected by HNC, there will never be one questionnaire moist suited to QOL reporting. The selection of questionnaires to be used in future studies of HNC patients should be based on several aspects, including hypothesis being tested (study objectives), questionnaire properties, and the potential benefits of combining different measures.

Even with this broad range of instruments, due to the complexity of HNC treatment and its potential physical, functional and emotional impairment, there are still areas of study not covered by the current questionnaires. Also, with the development of new treatment strategies and new treatment options, questionnaire modifications can be necessary, as the revised version of EORTC QLQ-H\&N35 which included items related to skin toxicities that could be found in patients submitted to targeted therapy $\left[5^{* *}, 9^{* *}\right]$.

In the Appendix there is a list of most QOL instruments used in patients with head and neck cancer.

\section{Limitations and challenges in QOL studies}

There are several limitations to be overcome in the QOL research. There are restrictions inherent in patient reported outcomes assessment, notably response shift, adaption, drop-out / responder bias and the wording and scoring of the questionnaires themselves. There is limitation in pooled data from different studies. Pooled data should enhance statistical power to identify differences when sub stratification by patient characteristics. Also, it allows meaningful comparisons across different treatment strategies and among different populations. This limitation is usually result of different methodologies utilized in the different published series, with different instruments, as well as due to differences in baseline data, mainly regarded to non clear information about co-morbidities and social habits, no clear methods of data collection and dropout rates and differences in treatment approaches.

Another limitation for comparison among different publications is the heterogeneous populations with limited number of patients included, mainly due 
to the lack of multi-institutional collaboration for QOL studies, reducing the number of participants which results in less robust conclusions.

It is important to note that cultural differences among populations living in different countries make general comparisons of results difficult to be understood. In this scenario, there is the necessity of cross-cultural validation of QOL instruments at different populations and cultures. Also, little is studied regarding the differences in the QOL evaluation among populations from developed and developing countries. In addition to differences related to staging at the time of diagnosis and to therapeutic strategies, religion, beliefs, cultural and social factors, as well as expectations must also be involved. In addition, the translation of a survey instrument into a second language is necessarily complex to guarantee its psychometric properties. In order to use a questionnaire produced and elaborated in another country, with a different language and culture, a process of translation and cultural adaptation, as well as psychometric validation is necessary, in order to obtain a reproducible, valid instrument with reliable results in the new population [16].

\section{Information derived from QOL studies and clinical practice}

Information derived from QOL studies has the potential to be incorporated in the clinical practice in several scenarios. Such information can be used for improvement in patient-physician relationship, communication and counseling $\left[5^{* *}, 17\right]$. The dissemination of QOL data can better equip patients to actively participate in the medical decisions concerning their care with the potential for improving the quality of care.

Some studies showed that information from QOL instruments can be clinical predictors of prognosis, as well as potential screening and planning tools for clinical care and rehabilitation efforts. The use of QOL measures can be used for assessing the need for further treatment, rehabilitation, relief symptoms, palliative care and social support. Practical examples: long-term complications as pain, depressive symptoms, dysphagia, and other issues usually not routinely assessed in the outpatient setting. 
The results of QOL studies in patients with HNC can be used in the treatment decision-making process. As described previously by one of the authors (SNR) [10], HRQOL has a role in treatment decisions when two treatments have a very good chance of cure with equivalent survival outcome but different HRQOL outcomes, when the HRQOL outcome is very poor and when the intention is noncurative - palliation 'QOL remaining time available'. Also, HRQOL can be considered an important additional factor in treatment decisions when there is a meaningful difference in HRQOL between two treatments but the HRQOL benefit is counter to the expected survival advantage (trade off).

\section{Tools to facilitate QOL information}

As a result of the evaluation QOL it is possible to present this data in a way that patients and cares can understand. It helps informed decision making, guide realistic expectations of outcome and helps patients get a better understanding of 'what will I be like' [18]. Although this resource is still in its infancy there is material available based on the UW-QOL for 26 subgroups based on early and late stage disease, site and treatment. In the future, with more data and refinement, this sort of web-based information can become more individualized and part of a holistic patient treatment programme.

Given the huge variation in what patients and their cares would like information on and support with, the other relative new extension to QOL assessment is the inclusion of a HNC specific item prompt list $\left[19^{*}, 20\right]$. This is a list of 56 issues divided into five domains: physical and functional well-being, treatment related concerns, social care and social well-being, psychological, emotional and spiritual well-being, and a free text 'other' section. There is a link between the type and number of items raised on the PCI and QOL. The issues that patients wish to talk about can act as a focus for more effective and efficient consultations, more targeted use of information material, and better use of support services. Interventions can be based on patient need.

\section{Future perspectives}


Wider access to the internet, increased availability of mobile phones, enhancements in computer technology will all allow for QOL data to be collected more easily and frequently. It can be tuned to the patients on responses, through computer adaptive technology, so focused at an individual level. Innovations in computing through icons, verbal prompts, and patients' choice in the number of items completed, will facilitate questionnaire completion even in those with minimum educational and IT experience. Translations of questionnaires will be readily available. Although some lower health economies globally will be reliant on paper QOL questionnaire, more will be able to use computers as a means of reporting. It will become more precise in reflecting the patients QOL outcome and concerns. QOL assessment will be part of the personalized medicine agenda and also the 'big data' initiative of pooled health outcomes informing and shaping evidenced based health strategies. Advances technology will allow the information to be available quickly and presented in a meaningful way, hence allowing it to be used real-time in clinical care. This will lead to the opportunity for better multiprofessional care and improved QOL outcomes and probably ultimately contribute to improved well-being and survival.

\section{Conclusion}

Information from QOL studies can be useful in several scenarios to improve the quality of care. They can serve as clinical predictors of prognosis, potential screening and planning tools for clinical care and rehabilitation efforts, and be used in the treatment decision-making process. Enhancements in computer technology will allow for QOL data to be used more easily and frequently.

\section{Key points:}

? Patients with malignant head and neck neoplasms can present changes in important functions related to the feeding, communication and social interaction, usually resulting in a negative impact on their QOL 
? The majority of QOL studies are performed through the application of specific questionnaires, which can be divided into three groups; generic questionnaires, disease-specific questionnaires, and symptom or treatment specific questionnaires

? Information derived from QOL studies has the potential to be incorporated in the clinical practice to improve the quality of care

\section{Acknowledgements}

None

\section{Financial support and sponsorship}

None

\section{Conflicts of interest}

None 
Appendix - Most common QOL instruments available for use in HNC patients

- SITE/DISEASE-SPECIFIC (HNC)

EORTC Quality of Life Head and Neck 35 [21]

Functional Assessment of Cancer Therapy - Head and Neck Subscale [22]

University of Washington HNC Questionnaire [23]

Performance Status Scale for H\&N Cancer [24]

H\&N Oncology Health Status Assessment [25]

- FUNCTION OR SYMPTOM-SPECIFIC INSTRUMENTS

\section{Appearance/disfigurement}

Derriford Appearance Scale 59 [26]

Derriford Appearance Scale 24 [27]

\section{Chewing/oral rehabilitation}

The Liverpool Oral Rehabilitation Questionnaire [28]

\section{Mucositis}

Oral Mucositis Daily Questionnaire [29]

Oropharyngeal Mucositis Quality of Life Scale [30] 


\section{Speech}

Self Evaluation of Communication Experiences after Laryngectomy [31]

Speech Handicap Index [32]

Voice Handicap Index [33]

Voice symptom scale [34]

VRQOL [35]

Voice Prosthesis Questionnaire [36]

\section{Swallowing}

Dysphagia Handicap Index [37]

MD Anderson Dysphagia Inventory [38]

Sydney Swallow Questionnaire [39]

Swallowing Quality of Life Measure [40]

\section{Shoulder}

The Neck Dissection Impairment Index [41]

Shoulder Disability Questionnaire [42]

Disability of the Arm, Shoulder, and Hand Questionnaire [43]

\section{Trismus}

Gothenburg Trismus Questionnaire [44] 


\section{Xerostomia}

Groningen Radiotherapy Induced Xerostomia Questionnaire [45]

Xerostomia-Related Quality of Life Scale [46]

Xerostomia Questionnaire (XQ) [47]

Xerostomia Inventory (XI) [48] 


\section{References}

1. Singh B. Rehabilitation and quality of life assessment in head and neck cancer. In: American Cancer Society atlas of clinical oncology: cancer of the head and neck. Edited by Shah J. London: BC Decker; 2001. pp. 467-77

2. Constitution of the World Health Organization. Am J Public Health Nations Health. 1946; 36(11):1315-1323.

3. Vartanian JG, Carvalho AL, Yueh B, et al. Long-term quality of life evaluation after head and neck cancer treatment in a developing country. Arch Otolaryngol Head Neck Surg 2004; 130:1209-13.

4. Rogers SN, Semple C, Babb M, Humphris G. Quality of life considerations in head and neck cancer: United Kingdom National Multidisciplinary Guidelines. J Laryngol Otol. 2016; 130(S2):S49-S52.

5. Licitra L, Mesía R, Keilholz U. Individualised quality of life as a measure to guide treatment choices in squamous cell carcinoma of the head and neck. Oral Oncol. 2016 Jan; 52:18-23.

** This review provides an overview of the HRQoL data available in head and neck cancer and discusses how this information can be used to individualize treatment and maximize the patient's daily living.

6. Parkar SM, Shah MN. A relationship between quality-of-life and head and neck cancer: A systemic review. South Asian J Cancer 2015; 4:179-82

7. WHO. WHO QOL Measuring Quality of Life. 1999; [cited 2015 Apr 24]. Available from: http://www.who.int/mental health/media/68.pdf

8. Morton RP, Izzard ME. Quality of life outcomes in head and neck cancer patients. World J Surg 2003; 27:884-9.

9. Rogers SN. Improving quality-of-life questionnaires in head and neck cancer. Expert Review of Quality of Life in Cancer Care. 2016; 1:1, 61-71 ** This paper discuss the benefits, limitations, and difficulties of using HRQoL questionnaires in clinical practice.

10. Rogers SN. Quality of life for head and neck cancer patients - has treatment planning altered? Oral Oncol 2009; 45:435-439

11. Gourin CG. Outcomes Measurement in Patients with Head and Neck Cancer. Curr Oncol Rep 2014; 16:376-382

12. Guedes RL, Angelis EC, Chen AY, et al. Validation and application of the M.D. 
Anderson Dysphagia Inventory in patients treated for head and neck cancer in Brazil. Dysphagia 2013; 28:24-32.

13. Ojo B, Genden EM, Teng MS, et al. A Systematic Review of Head and Neck Cancer Quality of Life Assessment Instruments. Oral Oncol 2012; 48:923937.

** This systematic review on HNC QOL instruments categorizes and reviews the properties of the instruments. Of the 710 articles that met the inclusion criteria, 57 used different head and neck-specific instruments of QOL.

14. Funk GF, Karnell LH, Smith RB, Christensen AJ. Clinical significance of health status assessment measures in head and neck cancer: what do quality-oflife scores mean? Arch Otolaryngol Head Neck Surg 2004; 130:825-9

15. http://www.handle-on-qol.com/Index.aspx.

** HaNDLE-on-QoL is an online searchable database of all papers published from 1982 to present on quality of life in head and neck cancer that have used questionnaires.

16. Sánchez D, Chala A, Alvarez A, et al. Psychometric Validation of the M. D. Anderson Symptom Inventory-Head and Neck Module in the Spanish Language. J Pain Symptom Manage. 2016 Jun;51(6):1055-61.

17. Velikova G, Booth L, Smith AB, et al. Measuring quality of life in routine oncology practice improves communication and patient well-being: a randomized controlled trial. J Clin Oncol. 2004;15;22(4):714-24

18. http://www.headandneckcancer.co.uk/For+patients/What+will+I+be+like. $\underline{\operatorname{aspx}}$

19. Rogers SN, El-Sheikha J, Lowe D. The development of a Patients Concerns Inventory (PCI) to help reveal patients concerns in the head and neck clinic. Oral Oncol. 2009;45:555-561. doi:10.1016/j.oraloncology.2008.09.004. * This is the first paper describing the development of a question prompt list specific to head and neck cancer.

20. Miller N, Rogers SN. A review of question prompt lists used in the oncology setting with comparison to the Patient Concerns Inventory. Eur J Cancer Care (Engl). 2016 Mar 14. doi: 10.1111/ecc.12489. [Epub ahead of print]

21. Bjordal K, Hammerlid E, Ahlner-Elmqvist M, et al. Quality of life in head and neck cancer patients: validation of the European Organizatin for Research 
and Treatment of Cancer Quality of Life Questionnaire - H\&N35. J Clin Oncol 1999; 17:1008-19

22. List MA, D'Antonio LL, Cella DF, et al. The Performance Status Scale for head and neck cancer patients and the Functional Assessment of Cancer Therapy - Head and Neck Scale: a study of utility and validity. Cancer 1996; 77:2294301.

23. Hassan SJ, Weymuller EA Jr. Assessment of quality of life in head and neck cancer patients. Head Neck 1993; 15:485-96.

24. List MA, Ritter-Sterr C, Lansky SB. A performance status scale for head and neck cancer patients. Cancer 1990;66:564-9

25. Funk GF, Karnell LH, Christensen AJ, Moran PJ, Ricks J. Comprehensive head and neck oncology health status assessment. Head Neck. 2003; 25:561-75

26. Klassen A, Newton J, Goodacre T. The Derriford Appearance Scale (DAS-59). Br J Plast Surg. 2001; 54:647-648

27. Carr T, Moss T, Harris D. The DAS24: a short form of the Derriford Appearance Scale DAS59 to measure individual responses to living with problems of appearance. Br J Health Psychol. 2005;10:285-98.

28. Pace-Balzan A, Cawood JI, Howell R, Lowe D, Rogers SN. The Liverpool Oral Rehabilitation Questionnaire: a pilot study. J Oral Rehabil. 2004; 31:609-17.

29. Stiff PJ, Erder H, Bensinger WI, et al. Reliability and validity of a patient selfadministered daily questionnaire to assess impact of oral mucositis (OM) on pain and daily functioning in patients undergoing autologous hematopoietic stem cell transplantation (HSCT). Bone Marrow Transplant. 2006; 37:393-401.

30. Cheng KK, Leung SF, Thompson DR, et al. New measure of health-related quality of life for patients with oropharyngeal mucositis: development and preliminary psychometric evaluation. Cancer. 2007; 15;109:2590-9.

31. Johansson M, Rydén A, Finizia C. Self evaluation of communication experiences after laryngeal cancer - a longitudinal questionnaire study in patients with laryngeal cancer. BMC Cancer. 2008 Mar 27;8:80.

32. Dwivedi RC, St Rose S, Roe JW, et al. First report on the reliability and validity of speech handicap index in native English-speaking patients with head and neck cancer. Head Neck. 2011; 33:341-348. 
33. Jacobson B, Johnson A, Grywalski C, et al. The voice handicap index (VHI): development and validation. Am J Speech-Lang Pathol. 1997; 6:66.

34. Deary IJ, Wilson JA, Carding PN, MacKenzie K. VoiSS: a patient-derived Voice Symptom Scale. J Psychosom Res. 2003; 54:483-9.

35. Hogikyan N, Sethuraman G. Validation of an instrument to measure voicerelated quality of life (V-RQOL). J Voice 1999; 13:557-569.

36. Kazi R, Singh A, De Cordova J, et al. Validation of a voice prosthesis questionnaire to assess valved speech and its related issues in patients following total laryngectomy. Clin Otolaryngol. 2006; 31:404-410.

37. Silbergleit AK, Schultz L, Jacobson BH, et al. The Dysphagia handicap index: development and validation. Dysphagia. 2012 Mar; 27:46-52.

38. Chen AY, Frankowski R, Bishop-Leone J, et al. The development and validation of a dysphagiaspecific quality-of-life questionnaire for patients with head and neck cancer: the M. D. Anderson dysphagia inventory. Arch Otolaryngol Head Neck Surg. 2001; 127:870-876.

39. Dwivedi RC, St Rose S, Roe JW, et al. Validation of the Sydney Swallow Questionnaire (SSQ) in a cohort of head and neck cancer patients. Oral Oncol. 2010; 46:e10-4.

40. McHorney C, Robbins J, Lomax K, et al. The SWAL-QOL and SWAL-CARE outcomes tool for oropharyngeal dysphagia in adults: III. Documentation of reliability and validity. Dysphagia 2002; 17:97-114.

41. Taylor RJ, Chepeha JC, Teknos TN, et al. Development and validation of the neck dissection impairment index: a quality of life measure. Arch Otolaryngol Head Neck Surg. 2002; 128:44-49.

42. Van der Heijden G, Leffers P, Bouter L. Shoulder disability questionnaire design and responsiveness of a functional status measure. J Clin Epidemiol. $2000 ; 53: 29-38$.

43. Hudak PL, Amadio PC, Bombardier C. The Upper Extremity Collaborative Group (UECG) Development of an upper extremity outcome measure: the DASH (disabilities of the arm, shoulder and hand) Am J Ind Med. 1996; 29:602-608.

44. Johnson J, Carlsson S, Johansson M, et al. Development and validation of the Gothenburg Trismus Questionnaire (GTQ). Oral Oncol 2012; 48:730-6. 
45. Beetz I, Burlage FR, Bijl HP, et al. The Groningen Radiotherapy-Induced Xerostomia questionnaire: development and validation of a new questionnaire. Radiother Oncol. $2010 ; 97: 127-31$

46. Henson BS, Inglehart MR, Eisbruch A, Ship JA. Preserved salivary output and xerostomia-related quality of life in head and neck cancer patients receiving parotid-sparing radiotherapy. Oral Oncol. 2001; 37:84-93.

47. Eisbruch A, Kim HM, Terrell JE, et al. Xerostomia and its predictors following parotid-sparing irradiation of head-and-neck cancer. Int J Radiat Oncol Biol Phys. 2001 Jul 1;50(3):695-704.

48. Thomson W, Williams S. Further testing of the xerostomia inventory. Oral Surg Oral Med Oral Pathol Oral Radiol Endod. 2000; 89:46-50. 